Homeland Security Systems Engineering

and Development Institute

The Homeland Security Systems Engineering and Development Institute (hereafter "HS SEDI" or "SEDI") is a federally funded research and development center (FFRDC) established by the Secretary of Homeland Security under Section 305 of the Homeland Security Act of 2002. The MITRE Corporation operates SEDI under the Department of Homeland Security (DHS) contract number HSHQDC-09-D-00001.

SEDI's mission is to assist the Secretary of Homeland Security, the Under Secretary for Science and Technology, and the DHS operating elements in addressing national homeland security system development issues where enterprise, lifecycle, and/or acquisition systems engineering expertise is required. SEDI also consults with other government agencies, nongovernmental organizations, institutions of higher education, and nonprofit organizations. SEDI delivers independent and objective analyses and advice to support systems development, decision making, alternative approaches, and new insight into significant acquisition issues. SEDI's research is undertaken by mutual consent with DHS and is organized by Tasks in the annual SEDI Research Plan.

This paper does not necessarily reflect official DHS opinion or policy.

This document has been publically released. Case Number 13-3051 


\title{
Geophysical and Operational System Performance Tool for the Detection of Clandestine Tunnels: Enhancement and Case Studies
}

\author{
Carol Christou ${ }^{1}$, J. Casey Crager ${ }^{1}$, Walter Kuklinski ${ }^{1}$, Eliot Lebsack ${ }^{1}$, David Masters ${ }^{2}$, Mark Munson, \\ and Weiqun $\mathrm{Shi}^{1}$ \\ ${ }^{1}$ The MITRE Corporation \\ Bedford, MA \\ wshi@mitre.org \\ ${ }^{2}$ The Department of Homeland Security, S\&T \\ Washington, D.C.
}

\begin{abstract}
Clandestine tunnels in the southwest border have posed a serious threat and are a growing concern for United States' national security. To address the challenges and the asymmetric threat they present, the Department of Homeland Security (DHS) Science and Technology Directorate (S\&T) is presently leading the development of the Geophysical and Operational System Performance Tool (GOSPT) that can be used to assist technology acquisition, mission planning, and sensor performance assessment. The GOSPT combines subsurface geoenvironmental data of the southwest border, geophysical models, and sensor physics to conduct tunnel detection system performance analysis. Its capabilities include physics based 1D, 2D and 3D high-fidelity numerical modeling and simulation of various sensor system/configurations in operational environments. False targets and clutter may also be added to the simulations to assess sensor performance under a variety of conditions. This paper describes several enhancements of the GOSPT, including using advanced spatial and temporal processing techniques, and geo-statistical modeling tools, to improve sensor detection performance characterization. In addition, a Java-based, fully integrated end-to-end sensor simulation environment was developed to allow interactive sensor performance prediction, providing an effective mechanism of exploring sensor system integration, hardware/software advancement and data fusion technology development. The tool enables system developers to quickly and precisely observe the effects on system performance to changes in sensor design and geological conditions. Case studies utilizing the GOSPT for assessing the performance of an existing ground penetrating radar system on a concrete-lined tunnel, and investigating potential improved detection capabilities using advanced sensor placement and coherent multiple element array technology will be discussed in the paper.
\end{abstract}

Keywords- tunnel detection, sensors, sensor performance, electromagnetic, GPR, seismic, homeland security, sensor fusion

\section{INTRODUCTION}

Clandestine tunnels in the southwest border have posed a

This work is is sponsored by the DHS S\&T Contract HSHQDC-12-J00172. Opinions and interpretations are those of the authors and are not necessarily endorsed by United States Government. serious threat and are a growing concern for United States' national security. The number of tunnels has increased dramatically over the past few years. They are used to smuggle illegal contraband and immigrants into and out of the United States. To address the challenges and the asymmetric threat they present, the Department of Homeland Security (DHS) Science and Technology Directorate (S\&T) is presently leading the development of the Geophysical and Operational System Performance Tool (GOSPT) that can be used to assist technology acquisition, mission planning, and sensor performance assessment. The GOSPT combines subsurface geo-environmental data of the southwest border, geophysical models, and sensor physics to conduct tunnel detection system performance analysis. The objective is to provide a physical basis for DHS to select the most capable system or combination of systems to operate in a specific area to detect and locate clandestine tunnels. The GOSPT provides a means of performance prediction for different types of subsurface sensing modalities for tunnels at a depth and configuration of the user's selection over a wide range of subsurface conditions. It is an aggregation of a variety of modeling and simulation software components and surface and sub-surface field data collections, which all perform in concert to provide a characterization of sensor performance. The development of the GOSPT and its utilities has been summarized in a previous paper [1]. This paper describes several enhancements of the GOSPT.

\section{GOSPT ARCHITECTURE}

The block diagram of the GOSPT is shown in Figure 1. The system consists of a number of relatively independent but closely associated components that affect the performance of tunnel detection sensors. The test scenarios are generated by integrated information from i) local and regional geology (subsurface structures and heterogeneities), ii) geography and geometry (tunnel depth, cross-section type, surface topography etc.) and iii) information of the sensor network (sensor locations and type). Simulation models employ modality 
specific signal processing methods to assess the achievable performance for the specified sensor modality and environmental conditions. The final product is the assessment of the likelihood of the detection of a tunnel or tunnels, represented by Signal to Noise Ratio (SNR), Probabilities of Detection (Pd), False Alarm (PFA), captured by a set of Receiver Operating Curves (ROC).

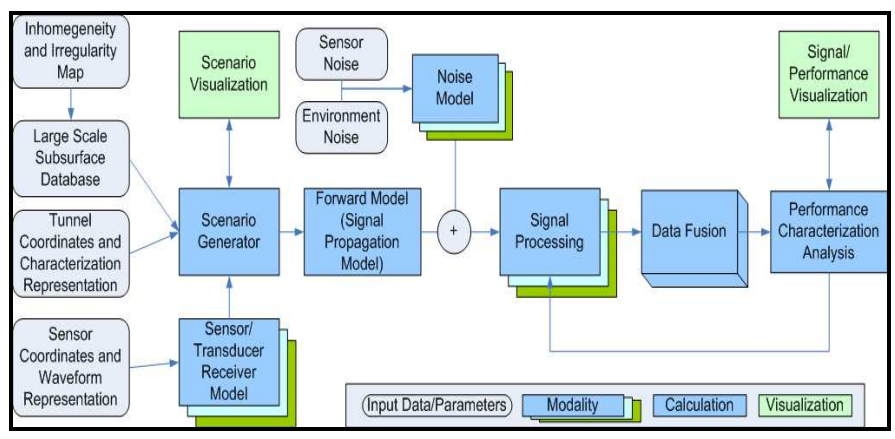

Figure 1: GOSPT block diagram.

\section{ADVANCED MODEL REPRESENTATION AND SIGNAL PROCESSING}

The GOSPT adapts a process to create realistic subsurface variation models. Given a nominal set of parameter data for a subsurface profile, it is important to account for the effects of random or deterministic variations of the geophysical parameters on the performance metrics. The GOSPT allows the implementation of both the deterministic and stochastic clutters. For random variations, a Fractional Brownian Motion (fBm) model [1] can be applied to enhance the subsurface model representations. Deterministic clutter may be incorporated in the form of discrete, concentrated inhomogeneities of varying position, shape, size and composition. To illustrate this utility, a realistic geologically plausible model was created for a concrete lined tunnel in a region of known near surface geology based on the photographs below (Figure 2). The three-dimensional (3D) $\mathrm{fBm}$ models simulating two tunnels (one is at $6 \mathrm{~m}$ depth and other one at $9 \mathrm{~m}$ ) are shown in Figure 3. Notice that the concrete liner has been included in the model, and based on the photos in Figure 2, the tunnel was estimated to be approximately $1 \mathrm{~m}$ wide by $2 \mathrm{~m}$ tall.

A popular $100 \mathrm{MHz}$ ground penetrating radar (GPR) was modeled to make measurements at the two tunnel depths.
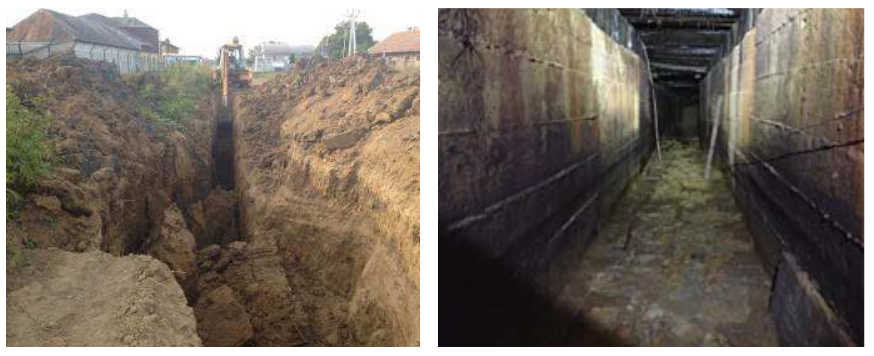

Figure 2: Photographs of soil (left) and concrete lined tunnel (right).
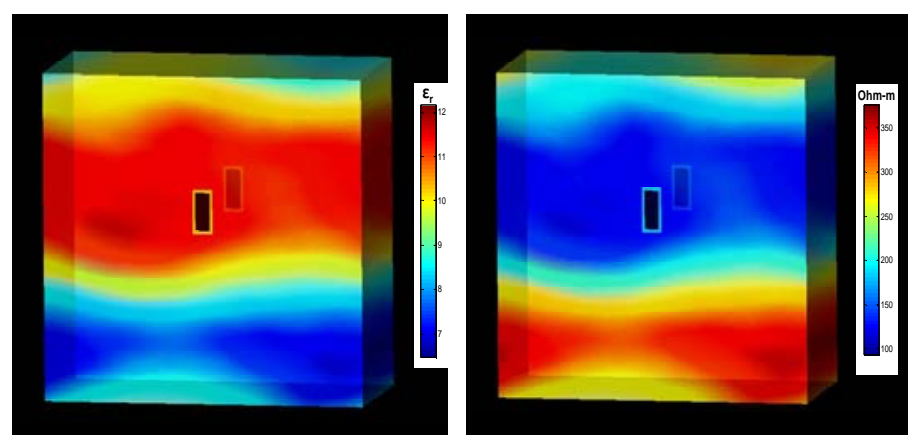

Figure 3: 3D fBm texture colored by relative permittivity (left) and resistivity (right), where the left colorbar extends from 6 (blue) to 12 (red), and the right colorbar extends from $90 \mathrm{Ohm}-\mathrm{m}$ (blue) to $376 \mathrm{Ohm}-\mathrm{m}$ (red). The concrete lined tunnel is shown at a depth of $6 \mathrm{~m}$.

To simulate the operation of the GPR, the detailed antenna system was modeled. The system consists of two collinear dipole antennas (one for transmit and the other for receive) separated by a distance of $2 \mathrm{~m}$. The transmit antenna was driven by a $100 \mathrm{MHz}$ Ricker pulse having a 100vol peak amplitude, and both antennas were modeled with $50 \mathrm{Ohm}$ source/load impedances. The simulated GPR took measurements every $8.56 \mathrm{~cm}$ over a strip of ground $21 \mathrm{~m}$ long, centered over the top of the tunnel. Simulations were performed with the tunnel at both 6 meter and 9 meter depths.

A user-specified cross-sectional slice of this 3D volume was then extracted for simulation using the two-dimensional (2D) electromagnetic (EM) finite difference time domain (FDTD) module of GOSPT. Using this subsurface model, a set of radargrams were produced and used for the performance analysis. A set of radargrams showing the response measured at the surface for a set of 170 antenna array positions spaced $8.56 \mathrm{~cm}$ apart for the cases with no tunnel, a tunnel at 6 meter depth and a tunnel at 9 meter depth are seen in Figure 4 . The tunnel response is clearly visible at both a 6 meter and 9 meter depth however the maximum amplitude of the tunnel response is approximately $26 \mathrm{~dB}$ smaller for the tunnel at 9 meters compared to the tunnel at 6 meters. To better mimic field measurement conditions additive $1.0 \mathrm{mV}$ standard deviation zero mean white Gaussian noise was added to the calculated return sensor signals. This amplitude was approximately one third of the peak amplitude response from the 6 meter depth tunnel. The corresponding simulated radargrams are seen in Figure 5.

In addition to the radargram data visualization, a $\underline{\text { Synthetic }}$ Aperture Radar (SAR) image visualization capability has been added to GOSPT to provide the user with a more intuitive visualization than an unprocessed radargram, confirming the performance predictions. The SAR image formation process made use of the GOSPT subsurface database to calculate the image response of the sensor for a given transmit and receive antenna position and an assumed point scatterer location. The resulting images for the $6 \mathrm{~m}$ and $9 \mathrm{~m}$ tunnel are seen in Figures 
6 and 7 respectively. As in the radargram data a logarithmic pseudocolor amplitude scale is used. The tunnel is clearly seen in the 6 meter image at the correct depth and with a more readily visualized width and height that can be inferred for the corresponding radargram data seen in the middle panel of Figure 5. The 9 meter tunnel still is not visually apparent in the corresponding SAR image in the right panel of figure 5, however.

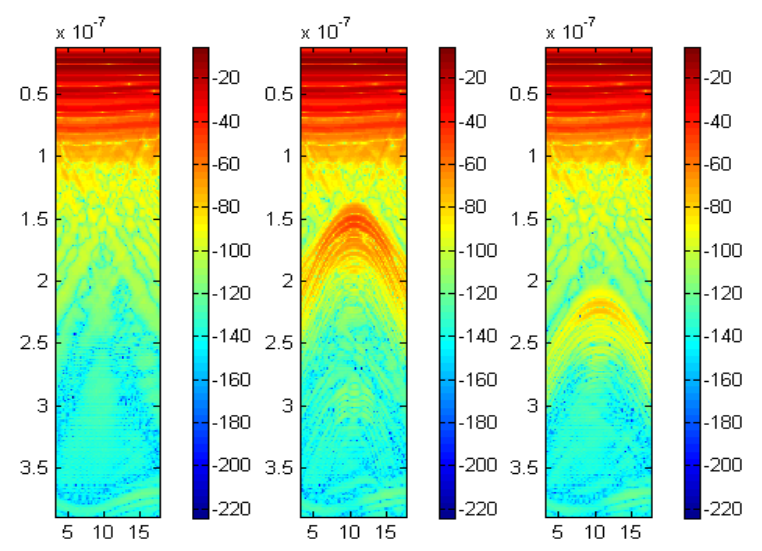

Figure 4: Radargrams for no tunnel (left), 6m depth tunnel (middle) and 9m depth tunnel (right). Pseudocolor response is in $\mathrm{dB}$ for time samples ( $\mathrm{y}$-axis) in seconds versus sensor position $(x-a x i s)$ in meters.
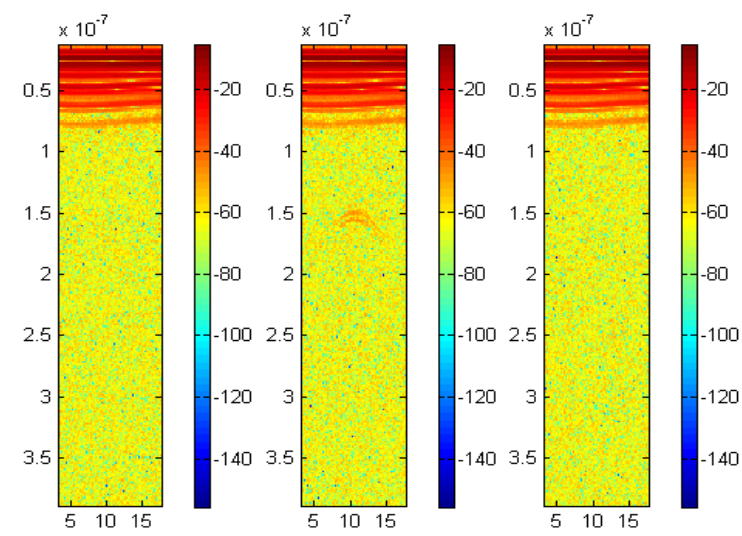

Figure 5: Radargrams for no tunnel (left), 6m depth tunnel (middle) and $9 \mathrm{~m}$ depth tunnel (right) with noise added. Pseudocolor response is in $\mathrm{dB}$ for time samples ( $\mathrm{y}$-axis) in seconds versus sensor position $(x$-axis) in meters.

\section{MATCHED FILTER DETECTION PROCESS}

The final product of the GOSPT is the assessment of the likelihood of the detection of a tunnel or tunnels, captured by a set of Receiver Operating Curves (ROC). A matched filter detector process has been adapted to generate the ROC curves.
Assume there are $N_{g}$ sensors arranged uniformly in a 1-D array closely coupled to the ground and denote the signals arriving at each sensor $n$ at time $t_{i}$ as $S_{n}\left(t_{i}, \vec{r}_{n}\right)$, where $i=1, .$. , denotes

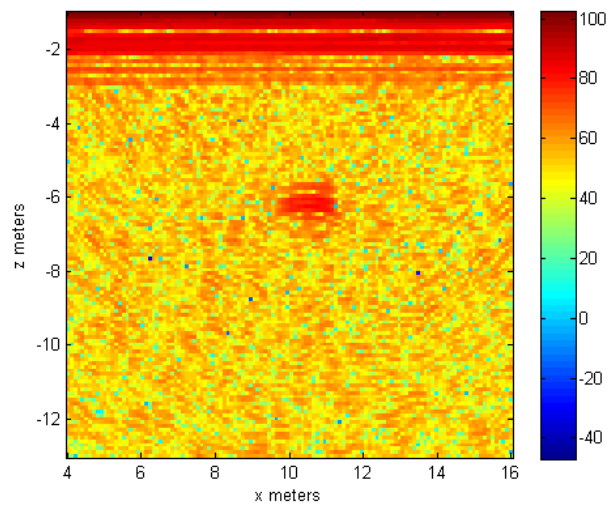

Figure 6: Subsurface SAR image for 6 meter depth tunnel.

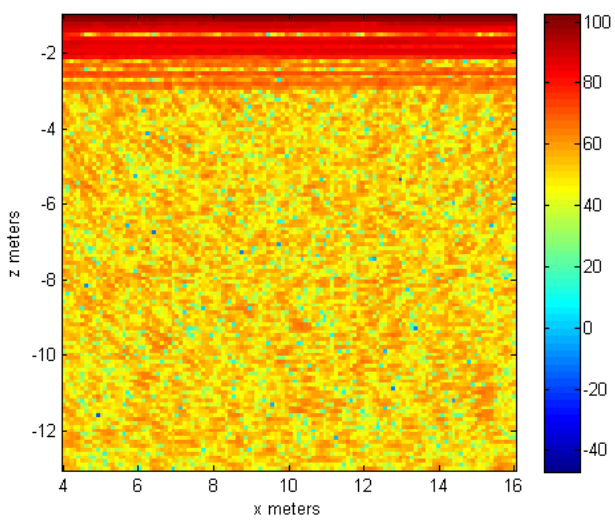

Figure 7: Subsurface SAR image for 9 meter depth tunnel.

the number of time steps $T$, and $\vec{r}_{n}$ is the position of the sensor on the surface in Cartesian coordinates with respect to a fixed reference point. For simplicity, assume that the sensor array is aligned along the $x$ direction, so that $\vec{r}_{n}=x_{n}$. Assume, furthermore, that $S_{n}^{T}\left(t_{i}, \vec{r}_{n}\right)$ is the detected signal with an underground tunnel present and $S_{n}{ }^{N T}\left(t_{i}, \vec{r}_{n}\right)$ is the signal without the presence of a tunnel. In signal processing, a matched filter (MF) is based on the correlation of an a priori known signal, or template, with an unknown signal to detect the correspondence of the template to the unknown signal. This is equivalent to a convolution of the unknown signal with a conjugate time-reversed form of the template. The matched filter is the optimal linear filter that maximizes the signal-tonoise ratio $(S N R)$ for additive noise. An overview of the method is shown in Figure 8.

The templates, shown in green, correspond to ensembles of signals received at the sensors for varying tunnel locations, sizes and shapes, and in principle can be arbitrarily many. Also, there is one template that corresponds to the signals received in the absence of a tunnel. After running the forward 
modeling code for a user-specified tunnel location in horizontal offset from the source and in depth, tunnel size and tunnel shape (rectangular or cylindrical), the sensor noise is added to the output signals, to which a Fourier Transform is then applied.

There are two hypotheses, $\mathrm{H}_{0}$ and $\mathrm{H}_{1}$, corresponding to the cases of no tunnel and tunnel present, respectively. Assuming a Gaussian distribution, the density function of $\ell_{i}$ under hypotheses $\mathrm{H}_{0}$ and $\mathrm{H}_{1}$ is:

$p_{\ell_{i} \mid 0,1}\left(\ell \mid H_{0,1}\right)=\frac{1}{\sqrt{2 \pi} \sigma_{\ell_{i}}} \exp \left(-\frac{\left(\ell-\ell_{i \mid 0,1}\right)^{2}}{2 \sigma_{\ell_{i}}{ }^{2}}\right)$;

where the variance $\sigma_{\ell i}$ is the same for both $\mathrm{H}_{0}$ and $\mathrm{H}_{1}$. The detection probability and false alarm probability become:

$$
\begin{aligned}
& P D(\gamma)=\sum_{i=1}^{N} \int_{\gamma}^{\infty} p_{\ell_{i}}\left(\ell \mid H_{1}\right) \prod_{k \neq i} F_{\ell_{k}}\left(\ell \mid H_{1}\right) d \ell ; \\
& \operatorname{PFA}(\gamma)=\sum_{i=1}^{N} \int_{\gamma}^{\infty} p_{\ell_{i}}\left(\ell \mid H_{0}\right) \prod_{k \neq i} F_{\ell_{k}}\left(\ell \mid H_{0}\right) d \ell ;
\end{aligned}
$$

where $\gamma$ represents a threshold, $N$ is the number of templates, and $F_{\ell k}\left(\ell \mid H_{0,1}\right)$ is the cumulative distribution function of $\ell_{k}$ under hypothesis $H_{0,1}$. The underlying assumptions in this analysis are that the signals are deterministic, the additive noise is Gaussian, and

$$
\int H_{i}(f) H_{i^{\prime}}(f) S_{n}(f) d f \approx 0 ; \quad i \neq i^{\prime}
$$

i.e., the $\ell_{i} s$ are uncorrelated, where $S_{n}(f)$ is the noise spectral power density.

For multiple sensors, the resulting $\ell_{i}$ s for each sensor signal are added together to form a composite $\ell_{i}^{C}$.

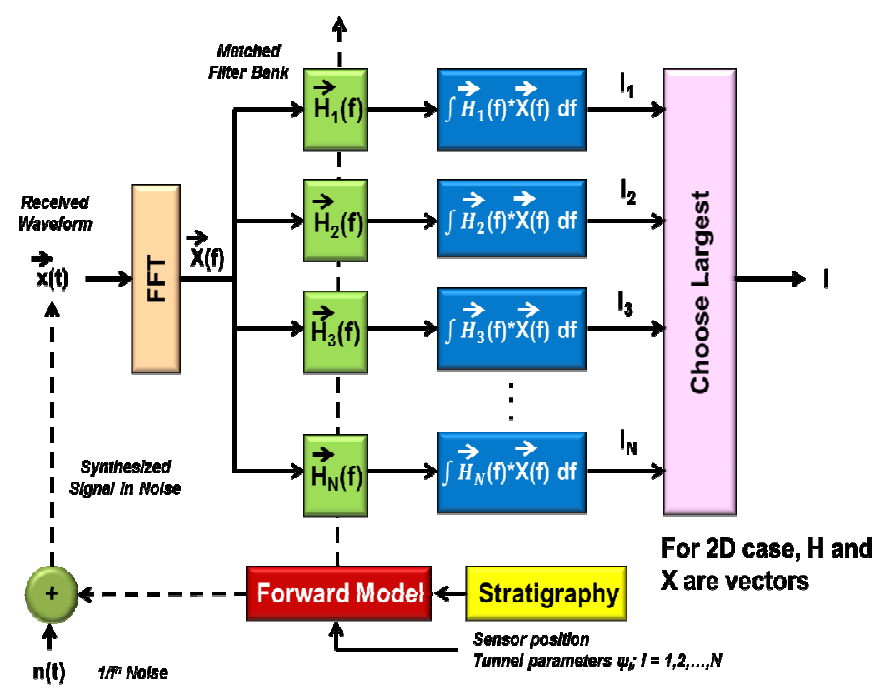

Figure 8. Overview of Matched Filter Detector
An example of the ROC curve is illustrated below for the seismic simulation module using the triaxial GS-11D rotating coil geophones, manufactured by GeoSpace Technologies, [2]. This type of sensors were used at a field data collection in an area of interest. Both the seismic compressional velocity $V_{p}$ and the shear wave velocity $V_{s}$, as well as attenuation factors $Q_{p}$ and $Q_{s}$ have been determined from the field measurements.

The three frequency response curves in Figure 9 correspond to three shunt damping levels. The geophone bandwidths are determined from the extent of the flat regions. The intrinsic sensitivity is $.810 \mathrm{~V} / \mathrm{in} / \mathrm{sec}$ according to the sensor specs. In addition to ambient noise, sensor noise must be taken into account when simulating the output of seismic measurements. Sensor self-noise was reported to be negligible for these particular geophones.

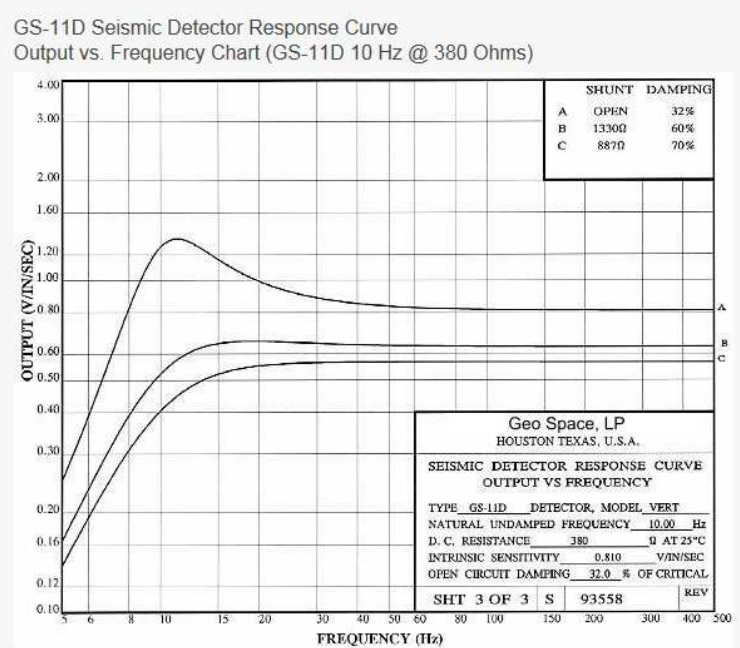

Figure 9: Frequency Response Curves for the GS-11D GeoSpace Geophone.

The seismic simulation was conducted using a MATLAB based 3D finite difference code [3]. The distribution of seismic noise was analyzed as a function of frequency using the reference data for 159 worldwide broadband stations [4]. The functional dependence on frequency was determined to vary according to $1 / f$ for velocity, with the spectrum becoming relatively flat at $f>1 \mathrm{~Hz}$. Time samples for each sensor were generated based on input seismic velocity and $Q$ factor profiles, frequency band and frontend bandpass filter characteristics. For 2-D input white noise $\left(T x N_{g}\right)$, an IIR filter is designed with the desired shape for seismic and equipment noise by determining the corresponding autoregressive coefficients for the noise process using the Yule-Walker algorithm. This algorithm provides a convenient recursion for generating these coefficients in a computationally efficient manner. The equipment related Brownian noise amplitude was assumed to be two orders of magnitude smaller than the environmental seismic amplitude. After adding ambient plus equipment noise to the output signal, a Butterworth filter is 
used to filter the total signal through the geophone bandwidth and a Fourier transform is applied. The returned signal frequency components are then multiplied by the corresponding components for each template, and the results summed over frequency. The largest output, $\max (\ell)$, corresponds to the likeliest template for the tunnel. One template corresponds to the case of no tunnel present.

A case with a $1 \times 2 \mathrm{~m}^{2}$ tunnel $10 \mathrm{~m}$ deep with $20 \mathrm{~m}$ offset from the source location is shown in Figure 10. The source was designed as a $50 \mathrm{~Hz}$ Ricker pulse with a bandwidth of $\sim 90 \mathrm{~Hz}$.

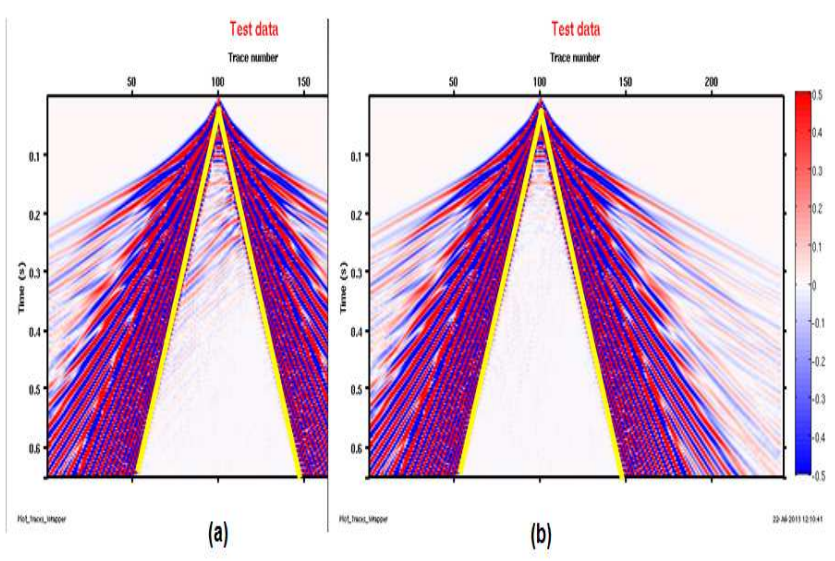

Figure 10: Seismograms for (a) tunnel present, (b) tunnel absent.

The corresponding ROC curves are shown in Figure 11for 7000 time steps and correspond to collective processing of signals received at 95 sensors around the source position.

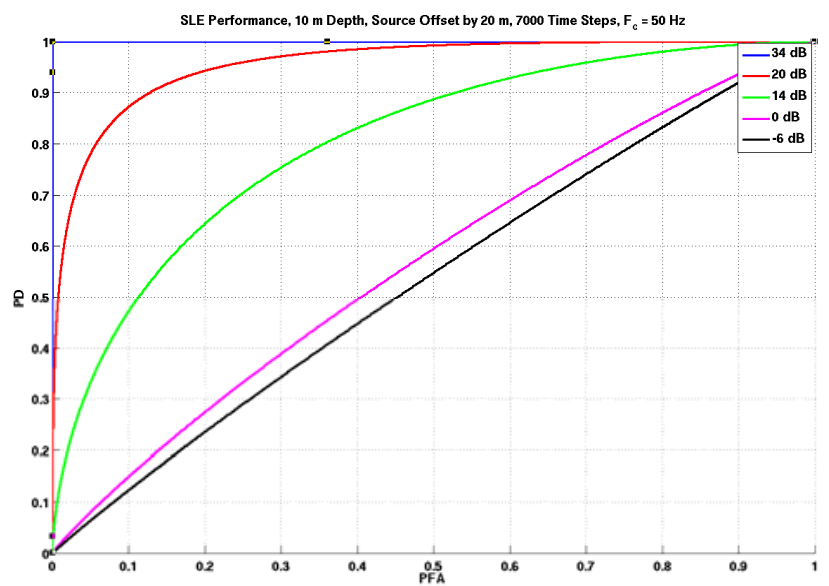

Figure 11: The ROC curves were obtained for SNR values of 34, 14, 0 and $-6 \mathrm{~dB}$, respectively

The ROC curves were obtained for SNR values of 34, 14,0 and $-6 \mathrm{~dB}$, respectively. As indicated the performance degrades as the signal-to-noise ratio decreases.

\section{SYSTEM INTEGRATION}

Capabilities of the GOSPT codebase have been enhanced through the utilization of a Java based service framework with an attached Graphical User Interface (GUI) display environment. Each of the major functional components shown in Figure 1 is implemented as orchestrated services which leverage both commercial and open source software libraries. When integrated together, the resulting framework provides a seamless end-to-end experience for investigating forward sensor models and their expected performance.

Development of the forward model scenario is facilitated through the use of a tabbed workflow interface. Figure 12 depicts a typical user workflow session prior to simulation execution. The Workflow process is enhanced through the inclusion of tabbed panels for hierarchical entity selection and interactive visualization of 3D Geophysical data. The user selects a subsurface region to define the area of interest, and introduces synthetic targets, as well as specifying forward model parameters via interaction with successive tabbed panels to create a working scenario.

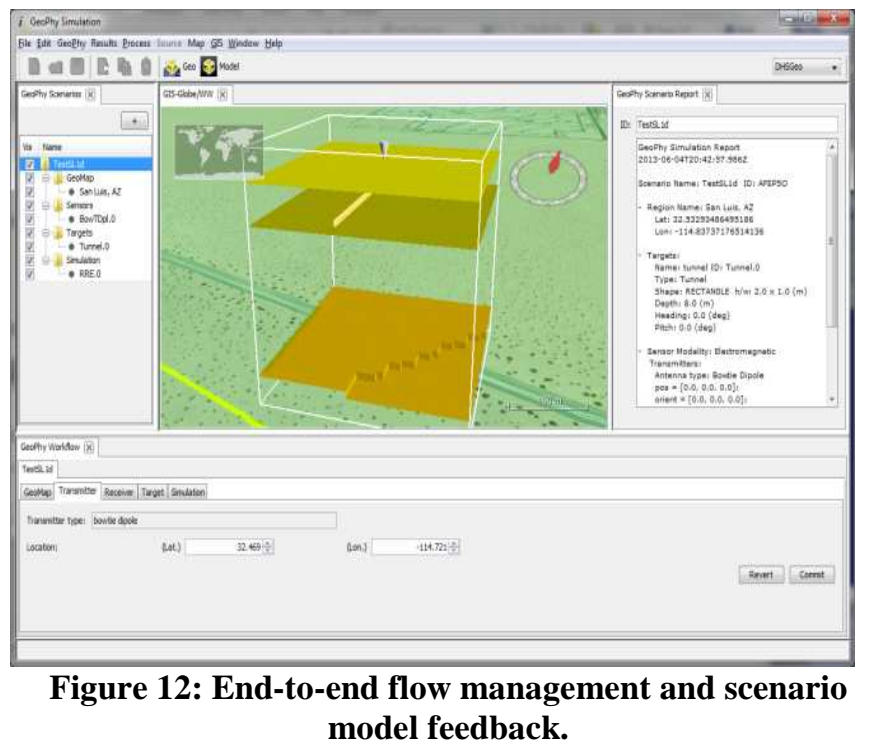

A complete simulation is composed of a forward model selection, synthetic targets, the geophysical background, and description of sensor placement and performance parameters. The various forward models (e.g. seismic, electromagnetic, etc.) are implemented as software components that act as attached services. These implementations are selectable by the user. For example, within the Electromagnetic family, selection options include 1D/2D/3D Finite Difference Time Domain (FDTD) for Ground Penetrating Radar (GPR). Once chosen, model attributes such as orientation, excitation waveform, and other relevant attributes may be adjusted prior to execution of the forward model definition. 
Forward model simulation may be performed via a contained execution of compiled MATLAB code, through direct execution of Java, or through a direct call to executable simulation code. The simulation interface provides a consistent mechanism for feeding forward model attributes into available simulations. Each simulation thread is managed via an asynchronous callback interface, which permits for interactive visual display presentation and capture of interim simulation products.

The Java GUI leverages NASA's World Wind display to provide In Situ subsurface attribute and feature visualization. As depicted in Figure IV-3, regions if the soil data are rendered as an elevated matrix of volumetric data. Selected subsurface geo-environmental data may be rendered at a location positioned over the earth's surface when sub-surface data is available.

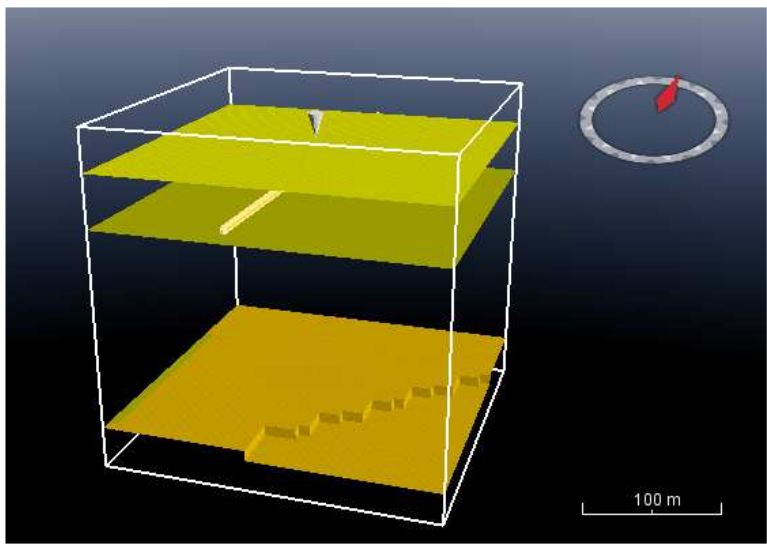

Figure 14: Subsurface geo-environmental data and synthetic tunnel.

As part of these analyses, it is important to have a noise model that spans the anticipated modalities. To this end, field collections of seismic and electromagnetic noise have been performed at selected locations within the areas encompassed by the geophysical spatial databases. It is understood that noise is often temporal in nature, and care has been taken to ensure that this behavior is captured in the noise model. The resulting noise spectra are then incorporated into the detection processing algorithms described previously to provide a reasonable expectation of subsurface structure detection performance.

Sensor-specific analysis products may also be produced, such as radargrams, seismograms, and displays of waveform propagation. Each product is tied to a forward model definition though use of a human readable hashing ID feature, which is associated with all display results. This hashing feature ensures that collections of results can be organized in a straightforward manner by the interface and the user.

\section{Conclusion}

The development of a physics-based approach to clandestine tunnel detection is necessary to estimate system performance, identify shortfalls in existing technologies, and make productive investments in research and development. Based on the effects of subsurface complexity and ambient noise on discriminating tunnels from sub-surface clutter, work will continue to refine the overall performance and utility of the GOSPT. Additional geological field collections, more forward modeling methods and new sensor modalities will be included as we continue sensor and geophysical modeling of the specific areas where the equipment will be operated.

\section{ACKNOWLEDGMENT}

We would like to thank Dr. John Lane and Carol Johnson from USGS Office of Groundwater in Storrs, CT, and Dr. Rich Miller from Kansas Geological Survey, for providing field data. We also give special thanks to Colin Shellum and Steve Nicely from DTRA's HTRAC Geotechnical Team for providing geological models

\section{REFERENCES}

1. C. Christou, J. C. Crager, L. Huffman, W. Kuklinski, E. Lebsack, D. Masters and W. Shi, " An Innovative method to determine multisystem performance for the detection of clandestine tunnels", 2012 IEEE Conference on Technologies for Homeland Security (HST) (pp. 43-50). Waltham, MA: IEEE, 2012.

2. GeoSpace Technologies, Calgary, Alberta, Canada, Geophones GS-11D, http://www.geospace.com/tag/gs-11d/, February, 2012.

3. Larsen, S., "E3D: 2D/3D ELASTIC FINITEDIFFERENCE WAVE PROPAGATION CODE . Retrieved 2011, from E3D: 2D/3D ELASTIC FINITE-DIFFERENCE WAVE PROPAGATION CODE Web Site: http://crack.seismo.unr.edu/ftp/pub/louie/class /455/e3d/e3d.txt, 1993.

4. D. McNamara, "Ambient Seismic Noise", www.mcnamara@usgs.gov, 2004. 